\title{
Comparison of computed tomography and magnetic resonance imaging in the evaluation of facet tropism and facet arthrosis in degenerative cervical spondylolisthesis
}

\author{
C. $\mathrm{Xu}^{1}$, Z.H. Ding ${ }^{1}$ and Y.K. Xu ${ }^{2}$ \\ 'Institute of Clinical Anatomy, Southern Medical University, Guangzhou, China \\ ${ }^{2}$ Department of Imaging Center, \\ Nanfang Hospital of Southern Medical University, Guangzhou, China \\ Corresponding authors: Z.H. Ding / Y.K. Xu \\ E-mail: zihaiding@163.com / yikaixu78@163.com
}

Genet. Mol. Res. 13 (2): 4102-4109 (2014)

Received May 9, 2013

Accepted October 15, 2013

Published May 30, 2014

DOI http://dx.doi.org/10.4238/2014.May.30.5

\begin{abstract}
The aim of this study was to determine the reliability of magnetic resonance imaging (MRI) in the assessment of facet tropism and facet arthrosis of spondylolisthesis levels in degenerative cervical spondylolisthesis as compared to computed tomography (CT). The discrepancies in the interpretation of CT and MRI data in the evaluation of facet tropism and arthrosis have given rise to questions regarding the reliability of comparisons of the two techniques. Using a 4-point scale, 3 blinded readers independently graded the severity of facet tropism and facet arthrosis of 79 cervical facet joints on axial T2-weighted and sagittal T1 and T2-weighted turbo spin echo images as well as the corresponding axial CT scans. All results were subjected to the kappa coefficient statistic for strength of agreement. In the assessment of the severity of facet arthrosis, intermethod agreement (weighted $\kappa$ ) between CT scanning with a moderate inter-rater reliability (range $\kappa=$ $0.43-0.57$ ) and MRI with fair inter-rater reliability (range $\kappa=0.23-0.38$ )
\end{abstract}


was 0.76 and 0.43 for the severity of facet tropism and facet arthrosis, respectively. Intra-rater reliability for the severity of facet arthrosis was moderate to substantial for CT and was moderate for MRI scans. Intrarater reliability for the severity of facet tropism was substantial to very good for CT and substantial for MRI scans. MRI can reliably determine the presence or degree of facet tropism but not facet arthrosis. Therefore, for a comprehensive assessment of cervical facet joint degeneration, both a CT and an MRI scan should be performed.

Key words: Computed tomography; Facet arthrosis; Facet tropism; Magnetic resonance imaging; Degenerative cervical spondylolisthesis

\section{INTRODUCTION}

Several studies in the early 1990s (Scotti et al., 1983; Breidahl et al., 1991; Bartlett et al., 1998; Dorenbeck et al., 2004) found that magnetic resonance imaging (MRI) could replace both computed tomography (CT) and CT-myelography as the primary imaging modality for the preoperative evaluation of patients with cervical radiculopathy, myelopathy, or both. Subsequently, MRI became the acceptable and recommended primary investigation tool for cervical radiculopathy, and MRI is currently widely used in the diagnosis of cervical disease in addition to all spinal abnormalities (Hesselink, 1988). Plain radiography (X-ray) and MRI are now the most common modalities used in the diagnosis and follow-up treatment of cervical degenerative spondylolisthesis, while the use of CT has declined in recent years.

In general, MRI provides more information than a CT scan. MRI is routinely used for the evaluation of spinal degenerative diseases due to its ability to accurately delineate soft-tissue structures (e.g., intervertebral discs, spinal ligaments, neural elements) as well as osseous structures (e.g., vertebral bodies, spinal canals, facet joints, uncovertebral joints). The ability of MRI to detect subtle abnormalities in both soft tissue and bone makes it highly sensitive in the detection of any pathological features (Yu and Williams, 2006); therefore, it has largely replaced plain CT as the primary screening tool for cervical degenerative disease (Brown et al., 1988).

In selecting the appropriate patient for surgery, the grading and classification of the facet arthropathy must be validated as part of the surgical planning. This requires communication between the spine surgeons and the radiologists prior to the surgical intervention. In our study, we compared the interpretation of CT and MRI images in the evaluation of the facet tropism and the severity of facet arthrosis of degenerative cervical spondylolisthesis. The purpose of this study was to establish concordance rates between the two techniques, identify the reasons for discordance, and determine if MRI is a reliable modality in the detection of facet arthropathy; if so, it could obviate the need for additional CT scans. To our knowledge, our investigation is the first to assess the reliability of CT and MRI in the detection of facet tropism and facet arthrosis of degenerative cervical spondylolisthesis.

\section{MATERIAL AND METHODS}

From January 2005 to August 2011, 54 patients were identified as having degenera- 
tive spondylolisthesis of the cervical spine in the absence of trauma, systemic inflammatory arthropathy, infection, neoplasia, or congenital abnormality. Patients with severe osteoporosis, incomplete patient data and records, or poor image quality were excluded from this study. The study subjects were retrospectively identified from a database of approximately 200 patients with surgically treated degenerative cervical spondylolisthesis. All patients examined had undergone both a CT and MRI scan of the cervical spine within 1 month of each other.

MRI was performed using a 1.5T Siemens Magnetom Vision (Siemens Corp., Germany). The following sequences were used for image acquisition with the surface coil: T1-weighted sagittal turbo spin-echo images (700/12/4/320/252x512:TR/TE/excitation/field of view acquisition matrix), T1-weighted axial conventional spin-echo images (588/17/2/250/256x256), axial gradient echo images (819/18/2/17/2/250/256x256/flip angle $30^{\circ}$ ), and T2-weighted sagittal turbo spin-echo images (5400/130/2/320/240x520). Slice thicknesses were determined using standard protocols and included T1-weighted sagittal images $(3 \mathrm{~mm})$, T1-weighted axial images $(4 \mathrm{~mm})$, gradient echo axial images $(4 \mathrm{~mm})$, and T2-weighted sagittal images $(3 \mathrm{~mm})$. Slices were contiguous without intervals. All CT scans was performed using GE LightSpeed CT scanners (Milwaukee, WI, USA). Standard clinical CT protocols were used for all examinations based on clinical indications. For spine CTs, a slice thickness of $2.5 \mathrm{~mm}$ and a tube voltage of $120 \mathrm{kV}$ were used. The tube current was automatically modulated along both the angular and $\mathrm{Z}$ directions based on body habitus from a preceding scanogram.

All MRI and CT images were evaluated independently by three experienced and blinded readers ( 1 spine surgeon, 1 musculoskeletal radiologist, and 1 musculoskeletal anatomist). They recorded the results with no prior clinical information or knowledge of the results of the other reader. They analyzed each individual cervical disc level and spinal canal for facet tropism and facet arthrosis to determine intra- and inter-reader reliability.

Using the method described by Noren et al. (1991) (Figure 1), the readers measured the facet angles to further classify the severity of facet tropism and facet arthrosis on a 4-point grading scale $(0=$ normal; I = mild; II = moderate; and III = severe) (Boden et al., 1996; Fujiwara et al., 1999) on both MRI and CT (Table 1, Figure 2).

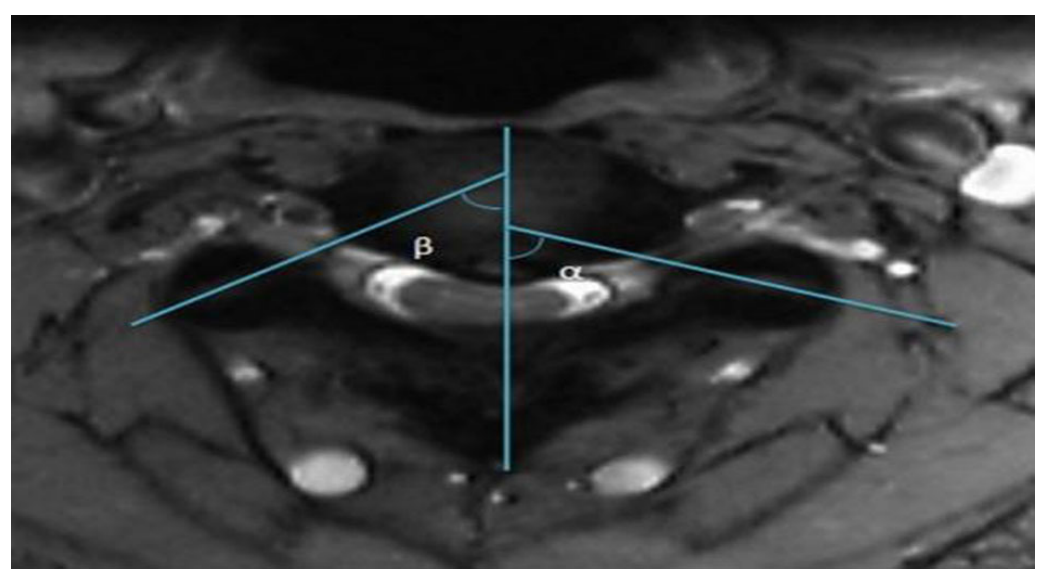

Figure 1. Illustration of the method used to measure facet joint angles on MRI. Here the intersection angle of the midsagittal line and the facet line is shown. 
Table 1. Criteria for grading facet arthrosis and facet tropism of the facet joints.

\begin{tabular}{|c|c|c|}
\hline Grade & Severity of facet tropism & Severity of facet arthrosis \\
\hline 0 (normal) & Absolute differences $\leq 6.00^{\circ}$ & Normal facet joint space (2-4 mm width) \\
\hline I (mild) & Absolute differences $6.01^{\circ}-10.00^{\circ}$ & $\begin{array}{l}\text { Narrowing of the facet joint space }(<2 \mathrm{~mm}) \text { and/or small osteophytes and/or mild } \\
\text { hypertrophy of the articular process }\end{array}$ \\
\hline II (moderate) & Absolute differences $10.01^{\circ}-16.00^{\circ}$ & $\begin{array}{l}\text { Narrowing of the facet joint space and/or moderate osteophytes and/or moderate } \\
\text { hypertrophy of the articular process and/or mild subarticular bone erosions }\end{array}$ \\
\hline III (severe) & Absolute differences $>16^{\circ}$ & $\begin{array}{l}\text { Narrowing of the facet joint space and/or large osteophytes and/or severe } \\
\text { hypertrophy of the articular process and and/or severe subarticular bone erosions } \\
\text { and/or subchondral cysts }\end{array}$ \\
\hline
\end{tabular}
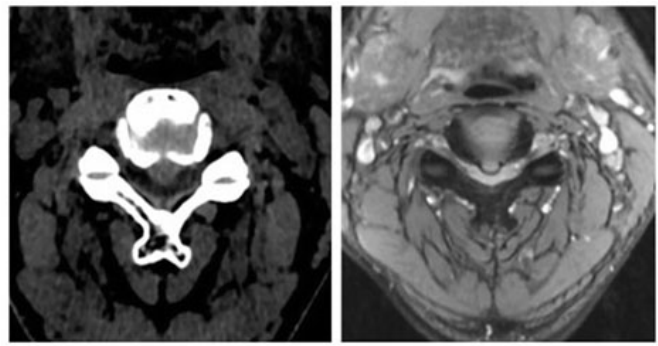

Grade 0
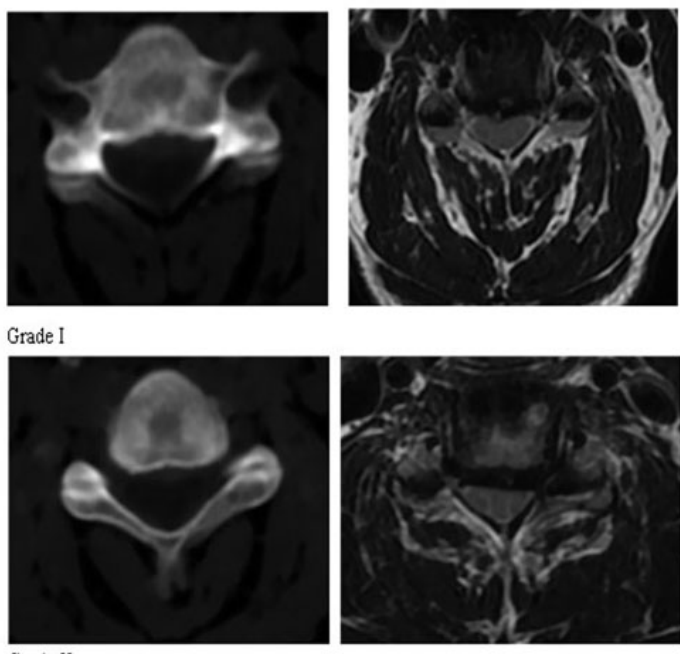

Grade II
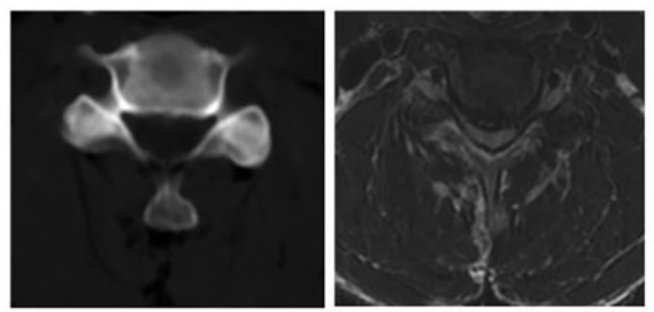

Grade III

Figure 2. Axial CT and MRI showing different degrees of facet arthropathy according to the Pathria classification. 
Statistical analysis was performed using the $\mathrm{k}$ statistic for strength of agreement. Weighted $\kappa$ values were used to evaluate the 4-point scale between CT and MRI as follows: 0 , chance agreement; 0 to 0.20 , poor agreement; 0.21 to 0.40 , fair agreement; 0.41 to 0.60 , moderate agreement; 0.61 to 0.80 , substantial agreement; 0.81 to 1.00 , very good agreement (Landis and Koch, 1977). Statistical analysis for calculations was performed using SPSS for Windows Version 13.0 (SPSS Inc., Chicago, IL, USA).

\section{RESULTS}

This investigation involved 54 patients ( 36 men and 18 women) with an average age of 52.6 years (range, $40-74$ years; median, 50 years). In the 54 subjects, 79 spondylolisthesis levels (C2-3 to C5-6) were evaluated, including 35 levels of C4-5, 22 levels of C5-6, 20 levels of $\mathrm{C} 3-4$, and 2 levels of C2-3. One hundred fifty-eight sites of interest were examined for each parameter evaluated.

\section{Inter-method agreement}

Using CT as the gold standard, we graded tropism of the facet joints as normal, mild, moderate, or severe. The weighted kappa coefficients for agreement between MRI and CT grading were 0.76 for the severity of facet tropism and 0.43 for the severity of facet arthrosis. MRI grading of facet tropism was identical to the CT grading in 134 of 158 joints (85\%), with substantial inter-method agreement. There was perfect agreement in grade 0 (42 of 158 joints) and poor agreement in grade II ( 28 of 158 joints). MRI grading of the severity of facet arthrosis was identical to the CT grading in 112 of 158 joints (71\%), with moderate inter-method concordance. There was perfect agreement in grade III ( 88 of 158 joints) and poor agreement in grades I and II ( 2 of 158 joints).

\section{Inter-rater reliability}

When assessing the severity of facet arthrosis, we found that CT performed better than MRI, which had moderate inter-rater reliability (range $\kappa=0.43-0.57$ ) and fair inter-rater reliability (range $\kappa=0.23-0.38$ ), respectively. However, for facet tropism, CT and MRI differed slightly, with substantial agreement in the inter-rater reliability (range $\kappa=0.66-0.78$ for CT and $\kappa=0.62-0.76$ for MRI).

\section{Intra-rater reliability}

The intra-rater reliability was higher than the inter-rater reliability for both CT and MRI. Intra-rater reliability for the severity of facet arthrosis was moderate to substantial with CT $(\kappa=0.67$ for reviewer $1, \kappa=0.57$ for reviewer 2 , and $\kappa=0.63$ for reviewer 3), and was moderate with MRI ( $\kappa=0.42$ for reviewer $1, \kappa=0.46$ for reviewer 2 , and $\kappa=0.41$ for reviewer 3 ). For facet tropism, intra-rater agreement was substantial to very good for CT $(\kappa=0.81$ for reviewer $1, \kappa=0.86$ for reviewer 2 , and $\kappa=0.77$ for reviewer 3$)$, and was substantial for MRI $(\kappa=0.79$ for reviewer $1, \kappa=0.75$ for reviewer 2 , and $\kappa=0.68$ for reviewer 3 ). 


\section{DISCUSSION}

Degenerative cervical spondylolisthesis is a common condition in the elderly, but it has received far less attention than degenerative lumbar spondylolisthesis (Jiang et al., 2011). One explanation could be that many patients are diagnosed with severe cervical spine deformities late in life, and when a deformity occurs, one does not necessarily conclude that spondylolisthesis was the primary cause (Woiciechowsky et al., 2004). Different theories exist about the mechanism of cervical spondylolisthesis. One is that it is caused by intervertebral disc degeneration and propagates via facet joints and ligaments (Lawhorne et al., 2009). Another theory is that, analogous to the lumbar spine, hypertrophic degeneration of the facet joints alters cervical mechanics and causes secondary spondylolisthesis (Deburge et al., 1995). An additional theory is that cervical degenerative spondylolisthesis results from an abnormal mechanical association between the intervertebral disc and facet joints, and then the facet joint and intervertebral disc degenerations occur together (Liu et al., 2006).

The cervical facet joints are the only synovial joints in the spine with hyaline cartilage overlying the subchondral bone. Facet tropism could cause facet joint degeneration and spine spondylolisthesis (Dai, 2001; Adams et al., 2006). Falco et al. (2012) proposed that facet arthrosis causes facetogenic pain, which leads to nape pain and facet tropism (Hall et al., 2010; Siegenthaler et al., 2010; Falco et al., 2012). The above findings indicate that both the detection and quantification of osteoarthritis of facet joints and facet tropism may aid in identifying cervical spondylolisthesis patients with nape pain (but no shoulder pain) and upper limb neurological symptoms, which would make them candidates for facet block therapy. In addition, the detection and quantification of facet arthrosis and facet tropism are both essential parts of a thorough presurgical evaluation of patients with cervical disc arthroplasty (Lehman et al., 2009).

Common diagnostic methods for the evaluation of facet joint degeneration are standard radiography, CT, and MRI (Haughton, 1995). Advanced CT and MRI procedures provide increased sensitivity, accurate anatomic detail, and cross-sectional images that can be reconstructed in different planes. It is generally accepted that CT captures excellent bone detail, whereas MRI is superior for the evaluation of soft-tissue structures such as bone marrow (Pooya et al., 2004; Whatmough and Lamb, 2006). In our retrospective investigation, we found that the inter-method agreement between CT and MRI with regard to facet arthrosis was moderate. Most disagreements were by grade I or II, whereas most agreements were by grade III. This indicates that the diagnosis of facet arthrosis using CT or MRI is similar. Our inter-method agreement of the two modalities of diagnosing facet arthrosis is lower than that reported by Weishaupt et al. (1999); however, it is similar to that reported by Lehman et al. (2009) and Shafaie et al. (1999). The inter-method agreement with regard to facet tropism was substantial, and MRI was identical to CT in the grading of 134 of the 158 cervical joints $(85 \%)$. This indicates that the diagnosis of facet tropism was the same for CT and MR imaging. Based on our findings, we believe that MRI should be augmented with $\mathrm{CT}$ for the assessment of facet joints in degenerative cervical spondylolisthesis. Our view is similar to that of Weishaupt et al. (1999) but not of Lehman et al. (2009). Lehman et al. (2009) proposed that the cervical spine is clearly different in anatomy and size, making MRI more difficult to use in the evaluation of facet disease. However, Weishaupt et al. (1999) view took into account that a significant proportion of the disagreements may be not due to the imaging methods themselves, but to the difficulties that readers might have in applying 
the grading system consistently.

In our retrospective analysis, we found that the intra-rater agreement was higher than the inter-rater agreement for both CT and MRI. In addition, the inter-rater and intra-rater agreements for CT were higher than for MRI for the grading of both facet arthrosis and facet tropism. Furthermore, inter-rater and intra-rater agreements for the grading of facet tropism were notably higher than for the grading of facet arthrosis. This suggests that MRI and CT were not significantly different in the evaluation of facet tropism. However, the difference in the evaluation of facet arthrosis between the two modalities was significant. Our results indicate that CT is indeed superior to MRI in the assessment of facet arthrosis. On the other hand, MRI can successfully be used to determine the orientation of the facet joint.

Although we did not investigate the role of the sagittal MRI images (T1WI and T2WI) in the grading of facet arthrosis, we did observe that all reviewers primarily used the axial plane for this purpose, and that the sagittal images were used only for confirmation of equivocal findings. In addition, unlike previously mentioned studies, our study used a spine surgeon, musculoskeletal radiologist, and musculoskeletal anatomist as reviewers because we wanted to investigate the concordance in the evaluation of facet arthropathy of degenerative cervical spondylolisthesis among these experts using CT and MR images.

\section{Limitations}

Weaknesses of our study consisted of the relatively few subjects, which limited our statistical power to detect true differences. Second, its retrospective nature and the use of CT scans as the gold standard may be considered as a limitation. Third, digital formatting and cursor-controlled measurements of the MRI and CT images on screen might potentially affect measurement errors and inter-rater reliability. Another potential bias in method concordance studies is the experience of the reviewers. All the readers in this study were very familiar with both imaging methods, and degrees of concordance would be expected to vary with levels of reviewer expertise.

\section{CONCLUSIONS}

In summary, our study found fair to moderate agreement between CT and MRI in the assessment of cervical facet arthrosis, and substantial to very good agreement in the assessment of cervical facet tropism. This suggests that MRI can reliably determine the presence or degree of facet tropism but not facet arthrosis. Therefore, in the assessment of facet joint degeneration, it is advisable to augment CT with MRI for an accurate diagnosis.

\section{Conflicts of interest}

The authors declare no conflict of interest.

\section{ACKNOWLEDGMENTS}

The authors would like to express their gratitude and great appreciation to all reviewers of the manuscript. There is no financial support to declare. 


\section{REFERENCES}

Adams MA, Bogduk N and Burton K (2006). The Biomechanics of Back Pain, 2nd edn. Elsevier Ltd, Philadelphia.

Bartlett RJ, Hill CR and Gardiner E (1998). A comparison of T2 and gadolinium enhanced MRI with CT myelography in cervical radiculopathy. Br. J. Radiol. 71: 11-19.

Boden SD, Riew KD, Yamaguchi K, Branch TP, et al. (1996). Orientation of the lumbar facet joints: association with degenerative disc disease. J. Bone Joint Surg. Am. 78: 403-411.

Breidahl WH, Low V and Khangure MS (1991). Imaging the cervical spine: a comparison of MR with myelography and CT myelography. Australas. Radiol. 35: 306-314.

Brown BM, Schwartz RH, Frank E and Blank NK (1988). Preoperative evaluation of cervical radiculopathy and myelopathy by surface-coil MR imaging. AJR Am. J. Roentgenol. 151: 1205-1212.

Dai LY (2001). Orientation and tropism of lumbar facet joints in degenerative spondylolisthesis. Int. Orthop. 25: 40-42.

Deburge A, Mazda K and Guigui P (1995). Unstable degenerative spondylolisthesis of the cervical spine. J. Bone Joint Surg. Br. 77: 122-125.

Dorenbeck U, Schreyer AG, Schlaier J, Held P, et al. (2004). Degenerative diseases of the cervical spine: comparison of a multiecho data image combination sequence with a magnetisation transfer saturation pulse and cervical myelography and CT. Neuroradiology 46: 306-309.

Falco FJ, Datta S, Manchikanti L, Sehgal N, et al. (2012). An updated review of the diagnostic utility of cervical facet joint injections. Pain Physician 15: E807-E838.

Fujiwara A, Tamai K, Yamato M, An HS, et al. (1999). The relationship between facet joint osteoarthritis and disc degeneration of the lumbar spine: an MRI study. Eur. Spine J. 8: 396-401.

Hall T, Briffa K and Hopper D (2010). The influence of lower cervical joint pain on range of motion and interpretation of the flexion-rotation test. J. Man. Manip. Ther. 18: 126-131.

Haughton V (1995). Imaging Techniques in Intraspinal Diseases. In: Diagnosis of bone and joint disorders. 3rd edn. (Resnick D, ed.). Saunders, Philadelphia, 237-276.

Hesselink JR (1988). Spine imaging: history, achievements, remaining frontiers. AJR Am. J. Roentgenol. 150: 1223-1229.

Jiang SD, Jiang LS and Dai LY (2011). Degenerative cervical spondylolisthesis: a systematic review. Int. Orthop. 35: 869-875.

Landis JR and Koch GG (1977). The measurement of observer agreement for categorical data. Biometrics 33: 159-174.

Lawhorne TW, III, Girardi FP, Mina CA, Pappou I, et al. (2009). Treatment of degenerative spondylolisthesis: potential impact of dynamic stabilization based on imaging analysis. Eur. Spine J. 18: 815-822.

Lehman RA, Jr., Helgeson MD, Keeler KA, Bunmaprasert T, et al. (2009). Comparison of magnetic resonance imaging and computed tomography in predicting facet arthrosis in the cervical spine. Spine (Phila Pa 1976) 34: 65-68.

Liu J, Ebraheim NA, Haman SP, Sanford CG Jr, et al. (2006). How the increase of the cervical disc space height affects the facet joint: an anatomy study. Spine (Phila Pa 1976) 31: E350-E354.

Noren R, Trafimow J, Andersson GB and Huckman MS (1991). The role of facet joint tropism and facet angle in disc degeneration. Spine (Phila Pa 1976) 16: 530-532.

Pooya HA, Seguin B, Tucker RL and Gavin PR (2004). Magnetic resonance imaging in small animal medicine: clinical applications. Comp. Cont. Educ. Pract. Vet. 26: 292-301.

Scotti G, Scialfa G, Pieralli S, Boccardi E, et al. (1983). Myelopathy and radiculopathy due to cervical spondylosis: myelographic-CT correlations. AJNR Am. J. Neuroradiol. 4: 601-603.

Shafaie FF, Wippold FJ, Gado M, Pilgram TK, et al. (1999). Comparison of computed tomography myelography and magnetic resonance imaging in the evaluation of cervical spondylotic myelopathy and radiculopathy. Spine (Phila Pa 1976) 24: 1781-1785.

Siegenthaler A, Eichenberger U, Schmidlin K, Arendt-Nielsen L, et al. (2010). What does local tenderness say about the origin of pain? An investigation of cervical zygapophysial joint pain. Anesth. Analg. 110: 923-927.

Weishaupt D, Zanetti M, Boos N and Hodler J (1999). MR imaging and CT in osteoarthritis of the lumbar facet joints. Skeletal Radiol. 28: 215-219.

Whatmough C and Lamb CR (2006). Computed tomography: principles and applications. Compend. Contin. Educ. Pract. Vet. 28: 789-800.

Woiciechowsky C, Thomale UW and Kroppenstedt SN (2004). Degenerative spondylolisthesis of the cervical spinesymptoms and surgical strategies depending on disease progress. Eur. Spine J. 13: 680-684.

Yu WD and Williams SL (2006). Spinal Imaging: Radiographs, Computed Tomography, and Magnetic Resonance Imaging. In: Orthopaedic Knowledge Update Spine. (Spivak JM and Connolly PJ, eds.). Rosemont, p. 57-68. 